\title{
USO DE CERA NA CONSERVAÇÃO PÓS-COLHEITA DO CAQUI CV. GIOMBO ${ }^{1}$
}

\author{
JULIUS BLUM ${ }^{2}$, FRANCINE BRODAY HOFFMANN ${ }^{3}$, RICARDO ANTONIO AYUB ${ }^{4}$, \\ DAIANA DE LOURDES JUNG ${ }^{5}$, MARCELO BARBOZA MALGARIM ${ }^{6}$
}

RESUMO-Este trabalho teve como objetivo avaliar a eficiência da cera de carnaúba na conservação pós-colheita do caqui cv. Giombo. Os tratamentos consistiram do tratamento- controle e rápida imersão nas soluções contendo 12,$5 ; 25$ e $50 \%$ do produto comercial Meghwax ECF $100^{\circledR}$, que é uma emulsão de cera de carnaúba não-iônica a $30 \%$. Após a secagem, os frutos foram armazenados a $4^{\circ} \mathrm{C}$ $\pm 1^{\circ} \mathrm{C}$ e $80 \%$ de umidade relativa. As avaliações foram realizadas em intervalos de 15 dias de conservação em câmara fria, seguidos de 4 dias à temperatura de $20 \pm 1^{\circ} \mathrm{C}$, simulando o período de comercialização. As variáveis analisadas foram: firmeza de polpa; sólidos solúveis; acidez titulável; $\mathrm{pH}$; teor de ácido ascórbico, fenóis e perda de massa fresca. O uso de cera de carnaúba, independentemente da concentração utilizada, diminuiu a perda de massa dos frutos em até 7,8\% em armazenagem por 60 dias em câmara fria, seguido de quatro dias em temperatura ambiente. A imersão dos frutos em solução com 12,5\% de cera foi eficiente na manutenção do teor de ácido ascórbico e da firmeza, prolongando o tempo de armazenamento por 6 dias. Com o decorrer do armazenamento, houve decréscimo da acidez e aumento do $\mathrm{pH}$.

Termos para indexação: Diospyrus kaki, pós-colheita, perda de massa.

\section{USE OF WAX IN ‘GIOMBO’ PERSIMMON COLD STORED}

ABSTRACT- This research had the objective of evaluating the efficiency of the "carnaúba" wax in post-harvest of persimmon fruits (Diospyros kaki), cv. Giombo. The treatments were the rapid immersion of fruits into solutions containing 12.5, 25 and $50 \%$ of the commercial product Meghwax ECF $100 \circledast$, being a $30 \%$ non ionic emulsion of carnaúba wax. After drying, fruits were stored at $4{ }^{\circ} \mathrm{C}$ and $80 \%$ RH. Chemical and physical characteristics of the fruits were measured throughout sixty days, at fifteen day intervals, followed by a 4-day period at $20^{\circ} \mathrm{C}$ simulating commercialization periods. The pulp firmness, $\mathrm{pH}$, soluble solids, titratable acidity, water loss, tannins and ascorbic acid contents were evaluated. The use of carnaúba wax, regardless of the concentration, diminished the loss of fresh mass of the fruits at up to $7.8 \%$ during storage periods of 60 days under cold chamber conditions, followed by a 4-day period at $20^{\circ} \mathrm{C}$. The immersion of the fruits into $12.5 \%$ of wax solutions was efficient to reduce losses of ascorbic acid and pulp firmness, extending therefore the storage period in 6 days. Throughout the time and ripening of the fruits, reductions of the acidity and increases of $\mathrm{pH}$ were also observed in the current study.

Index terms: Diospyrus kaki, post-harvest, loss of fresh mass.

O Brasil possui 8.322 ha plantados com caqui, com uma produção de 164.849 t e produtividade de $19.839 \mathrm{~kg} / \mathrm{ha}$ (Reetz et al., 2007), concentrando sua produção na cv. Fuyu, enquanto as outras cultivares apresentam projeção limitada. Entre estas cultivares, destaca-se o 'Giombo', bastante cultivada no Paraná e importante por ser uma variedade tardia, colhida em maio, escapando do período de grande oferta.

Visando a melhorar a conservação desta fruta, tem-se empregado a armazenagem em câmara fria, com reduzida eficiência, dados os problemas com injúria por frio (Fagundes et al., 2006). Outra opção é o uso da atmosfera controlada (Vidrih et al., 1990), mas com elevado custo de implantação. Ainda podem ser utilizados filmes plásticos para criar uma atmosfera modificada, estendendo a conservação de frutos por 48 dias de armazenamento em câmara fria, sem a ocorrência de escurecimento da epiderme (Tibola et al., 2005).

Os frutos naturalmente possuem ceras que reduzem a perda de água. Mas a aplicação de ceras modifica a composição interna dos gases na fruta, o que pode beneficamente reduzir a senescência ou levar à anaerobiose (Awad, 1993). A cera de carnaúba, uma palmeira brasileira, tem sido usada como revestimento em frutas e hortaliças, conferindo brilho e evitando as perdas por transpiração (Hagenmaier \& Baker, 1994). Por não ser tóxica, pode ser consumida nos frutos com casca e é facilmente removida com água.

Neste sentido, procurando-se alternativas de baixo custo

1(Trabalho 195-07). Recebido em: 13-08-2007. Aceito para publicação em: 28-03-2008.

${ }^{2}$ Eng. Agron. MSc. Bolsista da CAPES, Universidade Estadual de Ponta Grossa (UEPG), 84030-900 Ponta Grossa-PR. E-mail: juliusblum@yahoo.com.br ${ }^{3}$ Aluno do Curso de Agronomia, UEPG, 84030-900 Ponta Grossa-PR.

${ }^{4}$ Eng. Agron. Prof. Dr. do Departamento de Fitotecnia e fitossanidade, UEPG, Av. Carlos Cavalcante, 748, 84030-900 Ponta Grossa-PR. E-mail: rayub@uepg.br

${ }_{5}^{5}$ Aluno do Curso de Engenharia de Alimentos, UEPG, 84030-900 Ponta Grossa-PR.

${ }^{6}$ Eng. Agron. Dr. Pesquisador Instituto Agronômico do Paraná (IAPAR), Av. Presidente Kennedy, s/n Rod. do Café, Km 496, 84001-970 - Ponta GrossaPR.

Rev. Bras. Frutic., Jaboticabal - SP, v. 30, n. 3, p. 830-833, Setembro 2008 
que melhorem a conservação e apresentação de frutas, têm sido testados vários produtos que reduzam a transpiração, murchamento e enrugamento da casca que aumentem o brilho, como as ceras (Caron et al., 2003) e os biofilmes (Henrique \& Cereda, 1999; Vicentini et al., 1999). Estudos realizados com frutos de goiaba (Jacomino et al., 2003) e manga (Baldwin et. al., 1999) tratados com cera de carnaúba nas concentrações de 30 e 50\%, respectivamente, revelaram diminuição na perda de massa e podridões, e aumento no brilho dos frutos, prolongando o tempo de conservação.

Os tratamentos utilizados para prolongar a conservação pós-colheita de caquis visam a retardar a maturação do fruto. Durante a maturação, ocorrem algumas mudanças que podem ser avaliadas por métodos físico-químicos e são utilizadas como parâmetros para monitorar o progresso da maturação. A avaliação conjunta de parâmetros, como teor de sólidos solúveis, $\mathrm{pH}$, acidez e firmeza, serve para identificar o estágio de maturação em que se encontra a fruta, bem como acompanhar o processo de amadurecimento após a colheita.

Visando a evitar a perda de massa e firmeza de frutos de caqui, e conseqüentemente prolongar a conservação do fruto, o presente trabalho avaliou diferentes concentrações de cera de carnaúba na conservação frigorificada da cultivar 'Giombo'.

Os frutos de caqui, da cv. Giombo, provenientes de um pomar comercial, localizado no município de Porto AmazonasPR, foram colhidos no dia 09 de maio de 2007 e levados ao laboratório de Biotecnologia Aplicada a Fruticultura da Universidade Estadual de Ponta Grossa, para a aplicação dos tratamentos.

Os tratamentos foram constituídos pelo tratamentocontrole e imersão rápida dos frutos em solução contendo cera de carnaúba (Meghwax ECF $100^{\circledR}$ 30\%) diluída em água, nas concentrações de 12,5; 25 e $50 \%$ do produto comercial. Após a secagem da cera à temperatura ambiente, os frutos foram armazenados à temperatura de $4^{\circ} \mathrm{C} \pm 1^{\circ} \mathrm{Ce} 80 \%$ de umidade relativa por um período de 60 dias. Foram realizadas avaliações na data da aplicação dos tratamentos, e amostras dos frutos foram retiradas da câmara fria em intervalos de 15 dias e deixadas sobre bancada à temperatura de $20 \pm 1^{\circ} \mathrm{C}$, por quatro dias, simulando o período de comercialização, após o qual foram realizadas as análises.

As variáveis analisadas foram: firmeza de polpa $(\mathrm{N})$, utilizando penetrômetro com ponteira de $8 \mathrm{~mm}$, em dois pontos da zona equatorial dos frutos; Sólidos solúveis, determinados por refratometria expresso em ${ }^{\circ}$ brix; Acidez titulável por titulação com $\mathrm{NaOH}$ e resultado expresso em \% de ácido málico; $\mathrm{pH}$; Teor de ácido ascórbico (Eaton, 1989), teor de fenóis, através do método descrito por Singleton \& Rossi, (1965) e perda de massa fresca.

O experimento foi conduzido inteiramente ao acaso, com fatorial 4 × 5 ( 4 concentrações de cera x 5 períodos de armazenamento). A unidade experimental foi composta por 20 frutos, com 4 repetições. As médias dos tratamentos com cera foram comparadas pelo teste de Tukey, e o tempo de armazenamento foi analisado por regressão polinomial $(\mathrm{p} \leq 0,05)$.

A perda de peso acumulada foi linear com o passar do tempo nos frutos não-tratados, a equação de regressão revelanos uma perda de massa de $0,34 \%$ ao dia, resultando em uma perda total de 25,4\% aos 64 dias (Figura 1A). Ao final de 64 dias nos tratamentos com cera, independentemente da concentração, a perda de massa acumulada foi de aproximadamente 17,6 \%, revelando uma diminuição de perdas da ordem de $7,8 \%$ em relação à testemunha. A ausência de diferença da dose utilizada de cera na perda de peso também foi verificada por Ribeiro et al. (2005) em goiabas, testando as concentrações de 50 e $100 \%$ de cera.

A diminuição da perda de massa de frutos pela aplicação de cera é relatada freqüentemente na literatura (Oliveira \& Cereda, 2003; Jacomino et al., 2003; Ribeiro et al., 2005) e deve-se à baixa permeabilidade da cera ao vapor de água (Hagenmaier \& Baker, 1994). A redução da perda de água deve ser considerada o principal benefício da utilização da cera, visto que reduz perdas por murchamento do fruto, prolongando a vida de prateleira.

Ocorreu interação entre os tratamentos de cera e tempo de armazenamento para a firmeza de frutos. Houve redução quadrática em função do tempo para o tratamento de $12,5 \%$, e diminuição linear para os demais tratamentos (Figura 1B).

Segundo Rombaldi (1999), a firmeza recomendada para a comercialização do caqui deve estar acima de 20 N. Nesse trabalho, a firmeza foi o fator limitante na determinação do período de armazenagem dos frutos. O comportamento diferenciado da firmeza em função do tempo revelou-nos que o tratamento com $12,5 \%$ de cera manteve a firmeza acima de $20 \mathrm{~N}$ até 49 dias em câmara fria, enquanto os demais tratamentos mantiveram por apenas 43 dias, portanto o uso de cera na concentração de $12,5 \%$ prolongou a vida de prateleira dos caquis por 6 dias em comparação aos demais tratamentos. A manutenção da firmeza do fruto pelo uso da cera deve ser atribuída à maior turgescência e menor taxa respiratória do fruto, atrasando o processo de amadurecimento.

Houve interação entre os tratamentos à base de cera e o tempo de armazenamento para o teor de fenóis e ácido ascórbico (Figura 1C e D), apresentando comportamento semelhante com o tempo, com pequeno aumento nas concentrações até aproximadamente 20 dias, e queda acentuada nos últimos quinze dias de armazenagem, à exceção do tratamento com 12,5\% de cera, onde ocorreu menor diminuição nas concentrações dessas variáveis. Procurando-se verificar as relações entre as variáveis avaliadas, foi realizada análise de correlação (Pearson) entre atributos químicos e físicos dos frutos, revelando que a concentração de fenóis e ácido ascórbico tem relação positiva com a firmeza do fruto (Figura 2). Desse modo, ao passo que o fruto amadurece e perde a firmeza, os teores de fenóis e ácido ascórbico diminuem. Como o tratamento com 12,5\% de cera manteve os teores de ácido ascórbico e fenóis elevados por mais tempo, pode-se inferir que esse tratamento retardou o amadurecimento do fruto de caqui.

A elevação dos teores de ácido ascórbico nos primeiros dias de armazenamento também foi verificada por Ribeiro et al. (2005) para a goiaba, sendo este aumento relacionado à síntese de intermediários metabólicos precursores do ácido ascórbico, e a posterior diminuição à oxidação dos ácidos orgânicos durante o amadurecimento (Mercado-Silva et al., 1998). A diminuição do 
teor de ácido ascórbico e fenóis do fruto com o amadurecimento também é relatada por Antoniolli et al. (2000).

$\mathrm{O}$ uso de cera de carnaúba retardou o amadurecimento e conseqüente perda de fenóis, provavelmente pela diminuição da taxa respiratória do fruto.

Não houve interação entre tempo de armazenamento e concentração de cera para o $\mathrm{pH}$ e acidez dos frutos. Desse modo, os efeitos do tempo e tratamentos com cera serão apresentados e discutidos isoladamente. A acidez não foi alterada pela aplicação de cera de carnaúba, e o valor de $\mathrm{pH}$ foi mantido um pouco mais baixo no tratamento com 12,5\% de concentração (Tabela 1). Com o passar do tempo e amadurecimento dos frutos, ocorreu aumento do pH (Figura 1E) e diminuição da acidez (Figura 1F). A diminuição da acidez com o amadurecimento dos frutos também foi observada por Danieli et al.(2002), devido ao consumo de moléculas ácidas, principalmente ácidos orgânicos durante o processo de respiração.

O uso de cera de carnaúba diminuiu a perda de massa
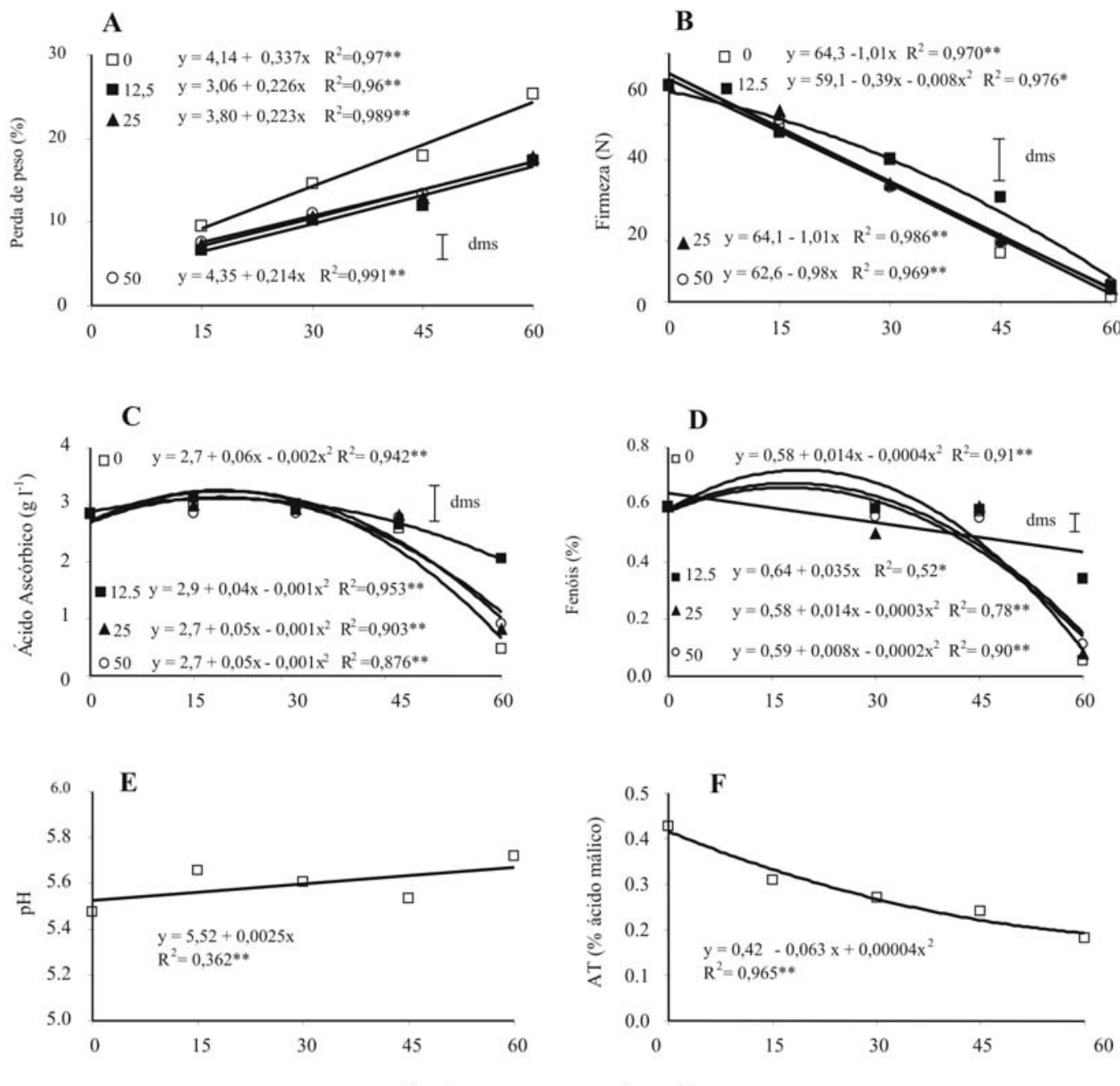

FIGURA 1- Firmeza (A), perda de peso (B), concentração de ácido ascórbico (C), fenóis (D), pH (E) e acidez (F) de frutos de caqui tratados com cera de carnaúba e armazenados a $4^{\circ} \mathrm{C}$. Nas Figuras E e F, cada ponto é média dos quatro tratamentos com cera. Ponta Grossa, 2007. * e ** Regressão polinomial significativa na probabilidade menor que 5 e 1 \% ,respectivamente.

dos frutos em 7,8 \% em armazenagem por 60 dias, em câmara fria, mais 4 dias em temperatura ambiente.

A imersão dos frutos em solução com $12,5 \%$ de cera de carnaúba foi eficiente na manutenção do ácido ascórbico e firmeza.

A conservação dos frutos de caqui foi prolongada com a imersão em solução com $12,5 \%$ de cera de carnaúba, permitindo o armazenamento por até 49 dias.

TABELA 1 - Efeito dos tratamentos com cera de carnaúba no $\mathrm{pH}$ e a acidez de frutos de caqui 'Giombo'. Valores médios de cinco épocas de amostragem. Ponta Grossa, 2007.

\begin{tabular}{lcc}
\hline Cera (\%) & pH & AT (\% ac. Málico) \\
\hline 0 & $5,62 \mathrm{ab}$ & $0,29 \mathrm{a}$ \\
12,5 & $5,56 \mathrm{c}$ & $0,29 \mathrm{a}$ \\
25 & $5,64 \mathrm{a}$ & $0,28 \mathrm{a}$ \\
50 & $5,57 \mathrm{bc}$ & $0,29 \mathrm{a}$ \\
\hline
\end{tabular}

Médias seguidas de uma mesma letra na coluna não diferem pelo teste de Tukey $(\mathrm{p} \leq 0,05)$.

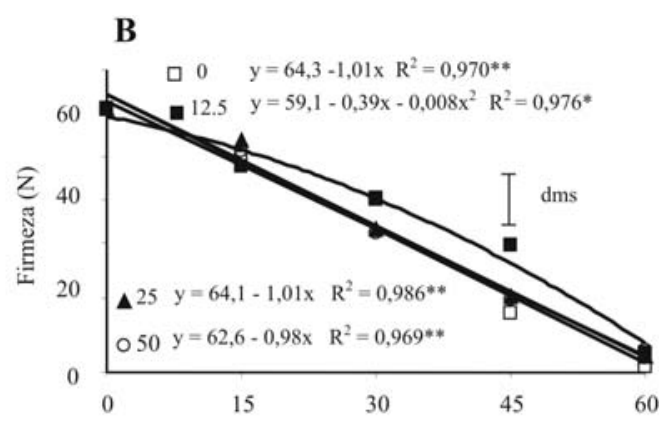



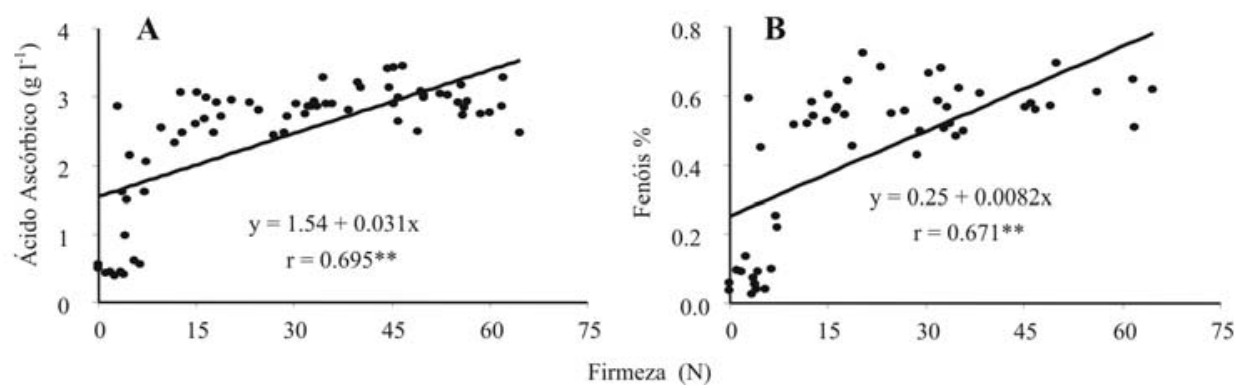

FIGURA 2 - Correlação da firmeza da polpa com a concentração de ácido ascórbico (A) e fenóis (B) de frutos de caqui Giombo, durante todo o período de amostragem dos frutos. Ponta Grossa, 2007. ** correlação significativa a 1\% de probabilidade.

\section{REFERÊNCIAS}

ANTONIOLLI, L. R.; CASTRO; P. R. C. KLUGE, R. A.; SCARPARE FILHO, J. A. Remoção da adstringência de frutos de caquizeiro 'Giombo' sob diferentes períodos de exposição ao vapor de álcool etílico. Pesquisa Agropecuária Brasileira, Brasília, v.35, n.10, p.2083-2091, out. 2000.

AWAD, M. Fisiologia pós-colheita de frutos. São Paulo: Nobel, 1993. 144p.

BALDWIN, E. A.; BURNS, J. K.; KAZOKAS, W.; BRECHT, J. K.; HAGENMAIER, R. D.; BENDER, R. J.; PESIS, E. Effect of two edible coatings with different permeability characteristics on mango (Mangifera indica L.) ripening during storage. Postharvest Biology and Technology, Amsterdan, v. 17, p. 215226, 1999.

CARON, V. N.; JACOMINO, A. P.; KLUGE, R. A. Conservação de cenouras 'Brasília " tratadas com cera. Horticultura Brasileira, Brasília, v. 21, n. 4, p. 597-600, 2003.

DANIELI, R.; GIRARDI, C. L.; PARUSSOLO, A.; FERRI,V. C.; ROMBALDI, C. V. Efeito da aplicação de ácido giberélico e cloreto de cálcio no retardamento da colheita e na conservabilidade de caqui, Fuyu. Revista Brasileira de Fruticultura, Jaboticabal, v. 24, n. 1, p. 044-48, 2002.

EATON, D. C. Laboratory investigations in organic chemistry. New York: McGraw-Hill, 1989.

FAGUNDES, A. F.; DABUL, A. N. G.; AYUB, R. A. Aminoethoxivinilglicina no controle do amadurecimento de frutos de caqui cv. Fuyu. Revista Brasileira de Fruticultura, Jaboticabal, v.28, n. 1, p. 73-75, 2006.

HAGENMAIER, R.D.; BAKER, R.A. Wax microemulsions and emulsions as citrus coating. Journal of Agricultural and Food Chemistry, Davis v.42, p.899-902, 1994.

HENRIQUE, C. M.; CEREDA, M. P. Utilização de biofilmes na conservação pós-colheita de morango (Fragaria ananassa Duch) cv. IAC Campinas. Ciência e Tecnologia de Alimentos, Campinas, v.19, n.2, p.205-210, 1999.
JACOMINO, A.P.; OJEDA, R. M.; KLUGE, R.A.; SCARPARE FILHO, J. A. Conservação de goiabas tratadas com emulsões de cera de carnaúba. Revista Brasileira de Fruticultura Jaboticabal, v.25, n.3, p.401-405, 2003.

MERCADO-SILVA, E.; BENITO-BAUTISTA, P.; GARCIAVELASCO, M.A. Fruit developmente, harvest index ande ripening changes of guavas produced in central México. Postharvest Biology na Technology, Wageningen, v.13, p.142-150, 1998.

OLIVEIRA, M. A.; CEREDA, M. P. Pós-colheita de pêssegos (Prunus pérsica L. Bastsch) revestidos com filmes à base de amido como alternativa à cera comercial. Ciência e Tecnologia de Alimentos, Campinas, v.23, p.28-33, 2003.

REETZ, E. R. Anuário brasileiro da druticultura. Santa Cruz do Sul: Gazeta, 2007. 136p.

RIBEIRO, V. G.; ASSIS, J.S. de; SILVA, F. F.; SIQUEIRA, P. P. X.; VILARONGA, C. P. P. Armazenamento de goiabas, 'Paluma' sob refrigeração e em condição ambiente com e sem tratamento com cera de carnaúba. Revista Brasileira de Fruticultura, Jaboticabal, v.27, n.2 p.203-206, 2005 .

ROMBALDI, C. V. Armazenamento de caqui. Jornal da Fruta, Lages, n. 232, p. 3, 1999.

SINGLETON, V. L.; ROSSI JÚNIOR, J. A. Colorimetry of total phenolics with phosphomolybidic-phosphotungstic acid reagents. American Journal Enology and Viticulture, Davis, v.16, p.144-158, 1965.

TIBOLA, C. S.; LUCCHETTA, L.; ZANUZO, M. R.; da SILVAP. R.; FERRI V. C.; ROMBALDI, C. V. Inibição da ação do etileno na conservação de caquis (Diospyrus kaki L.) 'Fuyu'. Revista Brasileira de Fruticultura, Jaboticabal, v.27, n. 1, p. 36-39, 2005.

VICENTINI, N. M.; CEREDA, M. P.; CÂMARA, F. L. A. Revestimento de fécula de mandioca, perda de massa e alteração da cor de frutos de pimentão. Scientia Agrícola, Piracicaba, v. 56, n. 3, p. 713-716, 1999.

VIDRIH, R.; SIMCIC, M.; HRIBAR, J. Storing of persimmon fruit under controlled atmosphere conditions. In: INTERNACIONAL HORTICULTURAL CONGRESS, 23., 1990, Firenze. Abstract... Firenze: ISHS, 1990.v.2, p.3312. 